\title{
Iterates of Markov Operators and Constructive Approximation of Semigroups
}

\author{
FrANCESCO ALtomARE*
}

\begin{abstract}
In this paper we survey some recent results concerning the asymptotic behaviour of the iterates of a single Markov operator or of a sequence of Markov operators. Among other things, a characterization of the convergence of the iterates of Markov operators toward a given Markov projection is discussed in terms of the involved interpolation sets.

Constructive approximation problems for strongly continuous semigroups of operators in terms of iterates are also discussed. In particular we present some simple criteria concerning their asymptotic behaviour.

Finally, some applications are shown concerning Bernstein-Schnabl operators on convex compact sets and Bernstein-Durrmeyer operators with Jacobi weights on the unit hypercube. A final section contains some suggestions for possible further researches.
\end{abstract}

Keywords: Markov operator, Iterate of Markov operators, Markov semigroup, Approximation of semigroups, Bernstein-Schnabl operator, Bernstein-Durrmeyer operator with Jacobi weights.

2010 Mathematics Subject Classification: 41A36, 47A35, 47D07.

\section{INTRODUCTION}

In this paper we survey some recent results concerning the asymptotic behaviour of the iterates of a single Markov operator or of a sequence of Markov operators.

Such problems are connected with ergodic theory and, in particular with ergodic theorems. Iterates of sequences of Markov operators are also involved in the constructive approximation of strongly continuous semigroups of operators and, hence, of the solutions to the initialboundary value differential problems governed by them.

Among other things, a characterization of the convergence of the iterates of Markov operators toward a given Markov projection is discussed in terms of the involved interpolation sets.

The usefulness of the approximation of strongly continuous semigroups of operators in terms of iterates, is enlightened by discussing some qualitative properties of them as well as their asymptotic behaviour.

Finally, some applications are shown concerning Bernstein-Schnabl operators on convex compact sets and Bernstein-Durrmeyer operators with Jacobi weights on the unit hypercube. A final section contains some suggestions for possible further researches. For more details about the results which are discussed in this paper we refer to [2], [4] and [5] and the references therein.

Received: 3 December 2018; Accepted: 23 December 2018; Published Online: 4 January 2019

*Corresponding author: F. Altomare; francesco.altomare@uniba.it

DOI: $10.33205 / \mathrm{cma} .491601$

Work partially supported by the Italian INDAM-GNAMPA. 


\section{PRELIMINARIES AND NOTATION}

Given a compact metric space $X$, we shall denote by $C(X)$ the linear space of all real-valued continuous functions on $X$ endowed with the supremum norm

$$
\|f\|_{\infty}:=\sup _{x \in X}|f(x)| \quad(f \in C(X))
$$

and the pointwise ordering, with respect to which it is a Banach lattice.

Let $\mathfrak{B}_{X}$ be the $\sigma$-algebra of all Borel subsets of $X$ and denote by $\mathcal{M}^{+}(X)\left(\right.$ resp., $\left.\mathcal{M}_{1}^{+}(X)\right)$ the subset of all Borel measures (resp. the subset of all probability Borel measures) on $X$.

The symbol $M^{+}(X)$ (resp., $M_{1}^{+}(X)$ ) designates the subset of all positive linear functionals on $C(X)$ (resp. the subset of all positive linear functionals $\mu: C(X) \rightarrow \mathbf{R}$ such that $\mu(\mathbf{1})=1, \mathbf{1}$ denoting the constant function with constant value 1).

By the Riesz representation theorem (see, e.g., [12, Section 29]), if $\mu \in M^{+}(X)$ (resp. $\mu \in$ $M_{1}^{+}(X)$ ), then there exists a unique (regular) Borel measure $\tilde{\mu} \in \mathcal{M}^{+}(X)$ (resp., in $\mathcal{M}_{1}^{+}(X)$ ) such that

$$
\mu(f)=\int_{X} f d \tilde{\mu} \quad \text { for every } f \in C(X) .
$$

Moreover, $\|\mu\|=\tilde{\mu}(X)$.

Consider a given Markov operator $T: C(X) \rightarrow C(X)$, i.e., $T$ is positive and $T(1)=1$. In the sequel a special role will be played by the subset of interpolation points of $T$ which is defined by

$$
\partial_{T} X:=\{x \in X \mid T(f)(x)=f(x) \text { for every } f \in C(X)\},
$$

and its possible representation by means of suitable functions.

Given a linear subspace $H$ of $C(X)$, its Choquet boundary $\partial_{H} X$ is the subset of all points $x \in X$ such that, if $\mu \in M^{+}(X)$ and if $\mu(h)=h(x)$ for every $h \in H$, then $\mu(f)=f(x)$ for every $f \in C(X)$.

If $H$ contains the constants and separates the points of $X$, then the Choquet boundary is non empty.

Given a Markov operator $T: C(X) \rightarrow C(X)$, we shall set

$$
M:=\{h \in C(X) \mid T(h)=h\} .
$$

Clearly, $M$ is contained in the range of $T$ which will be also denoted by

$$
H:=T(C(X))=\{T(f) \mid f \in C(X)\} .
$$

The subspace $M$ contains the constants and hence, if it separates the points of $X$, then its Choquet boundary $\partial_{M} X$ is not empty.

Theorem 2.1. Assume that the subspace $M$ defined above separates the points of $X$. Then

$$
\emptyset \neq \partial_{M} X \subset \partial_{T} X \subset \partial_{H} X .
$$

Moreover, if $V$ is an arbitrary subset of $M$ separating the points of $X$, then

$$
\partial_{T} X=\left\{x \in X \mid T\left(h^{2}\right)(x)=h^{2}(x) \text { for every } h \in V\right\} .
$$

Finally, if $\left(h_{n}\right)_{n \geq 1}$ is a finite or countable family of the linear subspace generated by $V$, separating the points of $X$ and such that the series $\Phi:=\sum_{n=1}^{\infty} h_{n}^{2}$ is uniformly convergent, then $\Phi \leq T(\Phi)$ and

$$
\partial_{T} X=\{x \in X \mid T(\Phi)(x)=\Phi(x)\} .
$$


For a proof of Theorem 2.1 we refer to [2, Theorem 2.1]) (see also [5, Theorem 1.3.1]).

As a particular case of the result above, consider a compact subset $X$ of $\mathbf{R}^{d}, d \geq 1$. For every $i=1, \ldots, d$ denote by $p r_{i}$ the $i^{t h}$ coordinate function on $X$, i.e., $p r_{i}(x):=x_{i}$ for every $x=$ $\left(x_{1}, \ldots, x_{d}\right) \in X$, and set

$$
\Phi_{d}:=\sum_{i=1}^{d} p r_{i}^{2}=\|\cdot\|^{2},
$$

where $\|\cdot\|$ stands for the Euclidean norm on $\mathbf{R}^{d}$.

Corollary 2.1. Given a Markov operator $T: C(X) \rightarrow C(X)$ such that $T\left(p r_{i}\right)=p r_{i}$ for every $i=1, \ldots, d$, then $\Phi_{d} \leq T\left(\Phi_{d}\right)$ and

$$
\partial_{T} X=\left\{x \in X \mid T\left(\Phi_{d}\right)(x)=\Phi_{d}(x)\right\} .
$$

\section{CONVERGENCE CRITERIA FOR NETS OF POSITIVE LINEAR OPERATORS}

In this section we discuss some general criteria concerning the convergence of nets (generalized sequences) of positive linear operators. The results seem to have an own independent interest and they can be considered as Korovkin-type theorems with respect to a limit operator which is an arbitrary positive linear operator rather then the identity operator. For additional Korovkintype theorems, we refer, e.g., to [1], [3] and the references therein.

For a given Markov operator $T: C(X) \rightarrow C(X)$, we proceed to state a criterion in terms of the subset $\partial_{T} X$ defined by (2.3), which concerns the convergence of nets of positive linear operators toward a positive linear operator $S: C(X) \rightarrow C(X)$ such that $S \circ T=S$.

In the subsequent section we shall use this result in order to investigate the asymptotic behaviours of iterates of Markov operators.

Theorem 3.2. Let $T: C(X) \rightarrow C(X)$ be a Markov operator such that the subset $\partial_{T} X$ is non empty and assume that there exists $\Psi \in C(X), \Psi \geq 0$, such that $\partial_{T} X=\{x \in X \mid \Psi(x)=0\}$ (for example $\Psi=T(\Phi)-\Phi$ as in Theorem 2.1).

Consider a net $\left(L_{i}\right)_{i \in I}^{\leq}$of positive linear operators from $C(X)$ into itself such that

(i) $\left(L_{i}(\mathbf{1}) \leq\right.$ is pointwise bounded (resp. uniformly bounded) on $X$.

(ii) $\lim _{i \in I} \leq L_{i}(\Psi)=0$ pointwise (resp., uniformly) on $X$.

Then, $\lim _{i \in I} \leq L_{i}(T(f)-f)=0$ pointwise (resp., uniformly) on $X$ for every $f \in C(X)$.

Accordingly, if $S: C(X) \rightarrow C(X)$ is a positive linear operator and if $L_{i}(T(f))_{i \in I}^{\leq}$converges pointwise (resp., uniformly) on $X$ to $S(f)$ for every $f \in C(X)$, then

$$
\lim _{i \in I} \leq L_{i}(f)=S(f)
$$

pointwise (resp., uniformly) on $X$ for every $f \in C(X)$. In particular, $S \circ T=S$.

As a special case of Theorem 3.2 we get the following Korovkin-type result.

Corollary 3.2. Let $T: C(X) \rightarrow C(X)$ be a Markov operator such that the subspace $M$ defined by (2.4) separates the points of $X$. Furthermore, set $H:=T(C(X))$ and consider $\Phi \in C(X)$ such that $\Phi \leq T(\Phi)$ and $\partial_{T} X=\{x \in X \mid T(\Phi)(x)=\Phi(x)\}$.

Given a Markov operator $S: C(X) \rightarrow C(X)$ such that $S \circ T=S$, if $\left(L_{i}\right)_{i \in I}^{\leq}$is a net of positive linear operators from $C(X)$ into itself and if $\lim _{i \in I} \leq L_{i}(h)=S(h)$ pointwise (resp., uniformly) on $X$ for every $h \in H \cup\{\Phi\}$, then $\lim _{i \in I} L_{i}(f)=S(f)$ pointwise (resp., uniformly) on $X$ for every $f \in C(X)$. 
The proofs of Theorem 3.2 and its subsequent Corollary 3.2 can be found in [2, Theorem 2.5 and Corollary 2.7]). Note also that it can be applied, e.g., for $S=T$ or for $S=\lambda T(\lambda \in C(X), 0 \leq \lambda)$ provided $T$ is a Markov projection, i.e. $T \circ T=T$.

An application of Corollary 3.2 will be shown in the subsequent Section 4 (see Theorem 5.8).

The next result can be useful to study the behaviour of nets of positive linear operators when the limit operator is unknown. It generalizes Theorem 2 of [19]. For its proof we refer to [10, Proposition 3.7]. In the same paper further remarks and applications can be found.

Let $(E,\|\cdot\|)$ be a Banach space of real-valued functions defined on a convex subset $X$ of a locally convex space. Assume that the space $E$, endowed with its norm and the pointwise order, is a Banach lattice.

Proposition 3.1. Let $\left(L_{i}\right)_{i \in I}^{\leq}$be a net of positive linear operators from $E$ into itself and assume that for every convex function $\varphi \in E$, the net $\left(L_{i}(\varphi)\right)_{i \in I}^{\leq}$is decreasing (resp., increasing).

Furthermore, assume that for some convex function $u \in E$, the net $\left(L_{i}(u)\right)_{i \in I}^{\leq}$is convergent in $E$. Then, setting

$$
\mathcal{A}(u):=\{g \in E \mid \text { There exists } \lambda \geq 0 \text { such that } \lambda u-g \text { and } \lambda u+g \text { are convex }\},
$$

the net $\left(L_{i}(f)\right)_{i \in I}^{\leq}$is convergent in $E$ for every $f \in \mathcal{A}(u)$.

Therefore, if $\mathcal{A}(u)$ is dense in $E$ and $\sup _{i \in I, i_{0} \leq i}\left\|L_{i}\right\|<+\infty$ for some $i_{0} \in I$, then $\left(L_{i}(f)\right)_{i \in I}^{\leq}$is convergent in $E$ for every $f \in E$.

Note that, if $X$ is a real interval and the convex function $u$ belongs to $C^{2}(X)$, then $\{f \in E \cap$ $C^{2}(X)|| f^{\prime \prime} \mid \leq \lambda u^{\prime \prime}$ for some $\left.\lambda \geq 0\right\} \subset \mathcal{A}(u)$. In particular, if $\alpha:=\min _{X} u^{\prime \prime}(x)>0$, then $\{f \in$ $E \cap C^{2}(X) \mid f^{\prime \prime}$ bounded $\} \subset \mathcal{A}(u)$, since $\left|f^{\prime \prime}\right| \leq \frac{\left\|f^{\prime \prime}\right\|}{\alpha} u^{\prime \prime}$ for every $f \in E \cap C^{2}(X)$, $f^{\prime \prime}$ bounded.

Moreover, if $\mathbf{1} \in E$ and a net $\left(L_{i}\right)_{i \in I}^{\leq}$satisfies the assumptions of Proposition 3.1, then the net $\left(L_{i}(\mathbf{1})\right)_{i \in I}^{\leq}$is constant. Therefore, if $E$ is a closed linear subspace of bounded continuous functions on $X$, equipped with the uniform norm, the net $\left(L_{i}\right)_{i \in I}^{\leq}$is equibounded as well.

If $X$ is a compact real interval, Proposition 3.1 applies in particular for $E=C(X)$ and $u \in$ $C^{2}(X)$ satisfying $\min _{X} u^{\prime \prime}(x)>0$.

Corollary 3.3. Given a compact real interval $X$, let $\left(L_{n}\right)_{n \geq 1}$ be a sequence of positive linear operators from $C(X)$ into itself and assume that for every convex function $\varphi \in C(X)$, the sequence $\left(L_{n}(\varphi)\right)_{n \geq 1}$ is decreasing (resp., increasing).

Further assume that for some convex function $u \in C^{2}(X)$ satisfying $\min _{X} u^{\prime \prime}(x)>0$, the sequence $\left(L_{n}(u)\right)_{n \geq 1}$ is uniformly convergent. Then for every $f \in C(X)$, the sequence $\left(L_{n}(f)\right)_{n \geq 1}$ is uniformly convergent.

For a multidimensional version of the above result we refer to [18, Theorem 2.2].

\section{Asymptotic BeHAVIOUR OF ITERATES OF MARKOV OPERATORS}

In this section we discuss some results concerning the asymptotic behaviour of iterates of Markov operators. For other additional results about this subject we refer, e.g., to [15, Theorem 1], [16, Theorem 3.1], [17, Theorem 1] and [23, Theorem 2.2].

Let $X$ be a compact metric space and consider two Markov operators $S: C(X) \rightarrow C(X)$ and $T: C(X) \rightarrow C(X)$ such that the subspace $M:=\{h \in C(X) \mid T(h)=h\}$ separates the points of $X$.

If $S \circ T=T$, then

$$
M \subset H:=T(C(X)) \subset\{h \in C(X) \mid S(h)=h\}
$$


and hence

$$
\emptyset \neq \partial_{M} X \subset \partial_{T} X \subset \partial_{H} X \subset \partial_{S} X .
$$

The proof of the next result is based on Theorem 3.2. For more details we refer to [2, Theorem 3.1]).

As usual, if $S: C(X) \rightarrow C(X)$ is a linear operator, the iterates $S^{n}, n \geq 1$, are defined recursively by $S^{1}:=S$ and $S^{n+1}:=S \circ S^{n}$.

Theorem 4.3. Let $S: C(X) \rightarrow C(X)$ and $T: C(X) \rightarrow C(X)$ be Markov operators and assume that the subspace $M:=\{h \in C(X) \mid T(h)=h\}$ separates the points of $X$. Then the following statements are equivalent:

(a) $\lim _{n \rightarrow \infty} S^{n}(f)=T(f)$ uniformly on $X$ for every $f \in C(X)$.

(b) $\lim _{n \rightarrow \infty} S^{n}(f)=T(f)$ pointwise on $X$ for every $f \in C(X)$.

(c) $S \circ T=T$ and $\partial_{S} X \subset \partial_{T} X$, i.e., for every $x \in X \backslash \partial_{T} X$ there exists $f \in C(X)$ such that $S(f)(x) \neq f(x)$.

(d) $S \circ T=T$ and for every sequence $\left(h_{n}\right)_{n \geq 1}$ in $M$ separating the points of $X$, such that the series $\Phi:=\sum_{n=1}^{\infty} h_{n}^{2}$ is uniformly convergent on $X$, one gets $\Phi \leq S(\Phi)$ and

$$
\{x \in X \mid S(\Phi)(x)=\Phi(x)\} \subset \partial_{T} X .
$$

(e) There exists $\Phi \in C(X)$ such that $\Phi \leq S(\Phi)$ and

$$
\{x \in X \mid S(\Phi)(x)=\Phi(x)\} \subset \partial_{T} X .
$$

Moreover, if one of the statements above holds true, then $T \circ S=T, T$ necessarily is a Markov projection, i.e., $T \circ T=T$, and

$$
\partial_{T} X=\partial_{S} X=\partial_{H} X
$$

It is not devoid of interest to point out that, if $T: C(X) \rightarrow C(X)$ is a Markov projection whose range separates the points of $X$, considering an arbitrary function $\Phi \in C(X)$ such that $\Phi \leq T(\Phi)$ and $\partial_{H} X=\{x \in X \mid T(\Phi)(x)=\Phi(x)\}$, then an example of a Markov operator $S: C(X) \rightarrow C(X)$ satisfying statement (c) of Theorem 4.3 is $S:=\lambda T+(1-\lambda) I$, where $I$ denotes the identity operator on $C(X)$ and $\lambda \in C(X)$ satisfies $0<\lambda(x) \leq 1$ for every $x \in X$. Below we show some applications of the results we have just described.

4.1. The Poisson operator associated with the classical Dirichlet problem. Consider a bounded open subset $\Omega$ of $\mathbf{R}^{d}, d \geq 2$, which we assume to be regular in the sense of potential theory (see, e.g., [3, Section 2.6]) and denote by $H(\Omega)$ the subspace of all $u \in C(\bar{\Omega})$ which are harmonic on $\Omega$.

Thus, for every $f \in C(\bar{\Omega})$ there exists a unique $u_{f} \in H(\Omega)$ such that $\left.u_{f}\right|_{\partial \Omega}=\left.f\right|_{\partial \Omega}$, i.e., $u_{f}$ is the unique solution to the Dirichlet problem

$$
\left\{\begin{array}{l}
\triangle u:=\sum_{i=1}^{d} \frac{\partial^{2} u}{\partial x_{i}^{2}}=0 \quad \text { on } \Omega, \quad\left(u \in C(\bar{\Omega}) \cap C^{2}(\Omega)\right) . \\
\left.u\right|_{\partial \Omega}=\left.f\right|_{\partial \Omega}
\end{array}\right.
$$

For instance, each bounded convex open subset of $\mathbf{R}^{d}$ is regular. Consider the Poisson operator $T: C(\bar{\Omega}) \rightarrow C(\bar{\Omega})$ defined by

$$
T(f):=u_{f} \quad(f \in C(\bar{\Omega}))
$$

The operator $T$ is a positive projection whose range is $H(\Omega)$. Moreover,

$$
\partial_{H(\Omega)} \bar{\Omega}=\partial_{T} \bar{\Omega}=\partial \Omega \text {. }
$$


A direct application of Theorem 4.3 gives the following result.

Corollary 4.4. Let $\Omega$ be a regular bounded open subset of $\mathbf{R}^{d}, d \geq 2$, and consider the Poisson operator $T: C(\bar{\Omega}) \rightarrow C(\bar{\Omega})$ defined by (4.6).

Furthermore, consider a Markov operator $S: C(\bar{\Omega}) \rightarrow C(\bar{\Omega})$ such that $S(u)=u$ for every $u \in H(\Omega)$ and assume that $\partial_{S} \bar{\Omega}=\partial \Omega$, i.e.,

$$
\text { for every } x \in \Omega \text { there exists } f \in C(\bar{\Omega}) \text { such that } S(f)(x) \neq f(x) \text {. }
$$

Then

$$
\lim _{n \rightarrow \infty} S^{n}(f)=T(f)
$$

uniformly on $\bar{\Omega}$ for every $f \in C(\bar{\Omega})$.

Moreover, combining Corollaries 3.2 and 2.1, we also get the following result which might be useful to approximate the Poisson operator.

Corollary 4.5. If $\left(L_{i}\right)_{i \in I}^{\leq}$is a net of positive linear operators from $C(\bar{\Omega})$ into itself and if $\lim _{i \in I} \leq L_{i}(h)=$ $T(h)$ pointwise (resp., uniformly) on $\bar{\Omega}$ for every $h \in H(\Omega) \cup\left\{\Phi_{d}\right\}$, then $\lim _{i \in I} \leq L_{i}(f)=T(f)$ pointwise (resp., uniformly) on $\bar{\Omega}$ for every $f \in C(\bar{\Omega})$.

4.2. Bernstein-Schnabl operators on convex compact subsets. Consider a metrizable convex compact subset $K$ (of some locally convex Hausdorff space) and denote by $A(K)$ the linear subspace of all real-valued continuous affine functions on $K$.

Consider a positive linear projection $T: C(K) \rightarrow C(K)$ such that

$$
A(K) \subset H:=T(C(K))=\{f \in C(K) \mid T(f)=f\}
$$

and

$$
h_{z, \alpha} \in H \text { for every } z \in K, \alpha \in[0,1], h \in H,
$$

where

$$
h_{z, \alpha}(x):=h(\alpha x+(1-\alpha) z) \quad(x \in K) .
$$

For instance, if $\Omega$ is a bounded open convex subset of $\mathbf{R}^{d}, d \geq 2$, then the Poisson operator defined by (4.6) is a positive projection satisfying (4.7) and (4.8).

We also recall that, if $K$ is a Bauer simplex (see, e.g., [3, Section 5.1] and [5, Section 1.1.3])(for instance, finite dimensional simplices are Bauer simplices), then there exists a unique positive linear projection $T: C(K) \rightarrow C(K)$ such that

$$
T(C(K))=A(K) .
$$

The projection $T$ is often referred to as the canonical positive projection associated with $K$. Actually, for every $f \in C(K), T(f)$ is the unique function in $A(K)$ that coincides with $f$ on the subset $\partial_{e} K$ of the extreme points of $K$. Clearly, $T$ satisfies (4.8) as well and

$$
\partial_{T} K=\partial_{e} K \text {. }
$$

In the finite dimensional case, considering the canonical simplex

$$
K_{d}:=\left\{\left(x_{1}, \ldots, x_{d}\right) \in \mathbf{R}^{d} \mid x_{i} \geq 0 \text { for every } i=1, \ldots, d \text { and } \sum_{i=1}^{d} x_{i} \leq 1\right\} .
$$


and setting $v_{0}:=(0, \ldots, 0), v_{1}:=(1,0, \ldots, 0), \ldots, v_{d}:=(0, \ldots, 0,1)$, then the canonical Markov projection $T_{d}: C\left(K_{d}\right) \longrightarrow C\left(K_{d}\right)$ associated with $K_{d}$, is defined by

$$
T_{d}(f)(x):=\left(1-\sum_{i=1}^{d} x_{i}\right) f\left(v_{0}\right)+\sum_{i=1}^{d} x_{i} f\left(v_{i}\right)
$$

$\left(f \in C\left(K_{d}\right), x=\left(x_{1}, \ldots, x_{d}\right) \in K_{d}\right)$.

When $K=[0,1]$, then the canonical projection is,indeed, the Markov operator $T_{1}: C([0,1]) \rightarrow$ $C([0,1])$ defined by

$$
T_{1}(f)(x)=(1-x) f(0)+x f(1)
$$

$(f \in C([0,1]), x \in[0,1])$.

For several other examples we refer to [3, Section 3.3] and [5, Chapter 3].

Coming back to a general positive linear projection $T: C(K) \rightarrow C(K)$ satisfying (4.7) and (4.8), let $S: C(K) \rightarrow C(K)$ be another positive linear operator such that

$$
S(h)=h \quad \text { for every } h \in H
$$

and

$$
\partial_{S} K=\partial_{T} K
$$

Let $\left(\tilde{\mu}_{x}^{S}\right)_{x \in K}$ be the unique family in $\mathcal{M}_{1}^{+}(K)$ such that

$$
S(f)(x)=\int_{K} f d \tilde{\mu}_{x}^{S} \quad(f \in C(K), x \in K) .
$$

For every $n \geq 1, x \in K$, and $f \in C(K)$, set

$$
B_{n}(f)(x)=\int_{K} \cdots \int_{K} f\left(\frac{x_{1}+\cdots+x_{n}}{n}\right) d \tilde{\mu}_{x}^{S}\left(x_{1}\right) \cdots d \tilde{\mu}_{x}^{S}\left(x_{n}\right) .
$$

By the continuity property of the product measure it follows that $B_{n}(f) \in C(K)$. Moreover, $B_{1}=S$.

The positive linear operator $B_{n}: C(K) \rightarrow C(K)$ is referred to as the $n$-th Bernstein-Schnabl operator associated with the positive linear operator $S$.

For special choices of the convex compact subset $K$ and of the operator $S$, these operators turn into the classical Bernstein operators on the unit interval, the unit $d$-dimensional hypercube and the $d$-dimensional simplex (see, e.g., [3, Section 6.1] and [5, Chapter 3]).

First, we point out that, by using a general Korovkin-type approximation theorem, it is possible to show that

$$
\lim _{n \rightarrow \infty} B_{n}(f)=f \text { uniformly on } K \text { for every } f \in C(K)
$$

(see [5, Theorem 3.2.1]).

Let $\left(u_{n}\right)_{n \geq 1}$ be an arbitrary (finite or countable) sequence in $A(K)$ separating the point of $\mathrm{K}$ and such that the series $\Phi:=\sum_{n=1}^{\infty} u_{n}^{2}$ is uniformly convergent on $K$.

Therefore, $\Phi \leq T(\Phi)$ and

$$
\partial_{H} K=\partial_{T} K=\{x \in K \mid T(\Phi)(x)=\Phi(x)\} .
$$

Accordingly, $B_{n}(\Phi)=\frac{1}{n} S(\Phi)+\frac{n-1}{n} \Phi$, so that, for every $x \in K$,

$$
B_{n}(\Phi)(x)-\Phi(x)=0 \text { if and only if } S(\Phi)(x)-\Phi(x)=0,
$$


i.e.,

$$
\partial_{T} X=\left\{x \in X \mid B_{n}(\Phi)(x)=\Phi(x)\right\}
$$

and $\Phi \leq B_{n}(\Phi)$. Thus all the assumptions of part $(e)$ of Theorem 4.3 are satisfied and hence we get that

Theorem 4.4. Under the preceeding hypotheses, for every $n \geq 1$,

$$
\lim _{m \rightarrow \infty} B_{n}^{m}(f)=T(f)
$$

uniformly on $K$ for every $f \in C(K)$.

In particular, for $n=1$,

$$
\lim _{m \rightarrow \infty} S^{m}(f)=T(f)
$$

uniformly on $K$ for every $f \in C(K)$.

The following special cases of Theorem 4.4 are worthy to be mentioned separately.

Corollary 4.6. Consider a metrizable Bauer simplex $K$ and denote by $T$ the canonical projection associated with $K$. Let $S: C(K) \rightarrow C(K)$ be a Markov operator such that $S(u)=u$ for every $u \in A(K)$ and $\partial_{S} K=\partial_{e} K$, i.e., for every $x \in K \backslash \partial_{e} K$ there exists $f \in C(K)$ such that $S(f)(x) \neq f(x)$.

Denoting by $\left(B_{n}\right)_{n \geq 1}$ the sequence of Bernstein-Schnabl operators associated with $S$, then for every $n \geq 1$,

$$
\lim _{m \rightarrow \infty} B_{n}^{m}(f)=T(f)
$$

uniformly on $K$ for every $f \in C(K)$. In particular, for $n=1$,

$$
\lim _{m \rightarrow \infty} S^{m}(f)=T(f)
$$

uniformly on $K$ for every $f \in C(K)$.

Corollary 4.7. Consider the Markov projection $T_{1}: C([0,1]) \rightarrow C([0,1])$ defined by (4.11). Let $S: C([0,1]) \rightarrow C([0,1])$ be a Markov operator such that $S(1)=1, S\left(e_{1}\right)=e_{1}$ and $\partial_{S}[0,1]=\{0,1\}$ i.e., for every $x \in] 0,1[$ there exists $f \in C([0,1])$ such that $S(f)(x) \neq f(x)$.

Denoting by $\left(B_{n}\right)_{n \geq 1}$ the sequence of Bernstein-Schnabl operators associated with $S$, then for every $n \geq 1$,

$$
\lim _{m \rightarrow \infty} B_{n}^{m}(f)=T_{1}(f)
$$

uniformly on $[0,1]$ for every $f \in C([0,1])$. In particular, for $n=1$,

$$
\lim _{m \rightarrow \infty} S^{m}(f)=T_{1}(f)
$$

uniformly on $[0,1]$ for every $f \in C([0,1])$.

Remark 4.1. Corollaries 4.6 and 4.7 apply in particular when, respectively, $K=K_{d}$ and $S=T_{d}$, $d \geq 1$ (see (4.10)) and when $K=[0,1]$ and $T=T_{1}$. In these cases, the corresponding BernsteinSchnabl operators are, indeed, the classical Bernstein operators on $K_{d}$ and on $[0,1]$.

In addition to the previous results, it is possible to investigate the limit behaviour of the iterates of Bernstein-Schnabl operators associated with a Markov projection even when the order of iteration depend of $n \geq 1$. The proof of the next result relies on Corollary 3.2 (see also [3, Theorem 6.1.3] or [5, Theorem 3.2.10]). 
Theorem 4.5. Let $T: C(K) \rightarrow C(K)$ be a positive Markov projection satisfying (4.7) and (4.8), and consider the relevant sequence $\left(B_{n}\right)_{n \geq 1}$ of Bernstein-Schnabl operators defined by (4.14) (with $\left.S=T\right)$. If $f \in C(K)$ and if $(k(n))_{n \geq 1}$ is a sequence of positive integers, then

$$
\lim _{n \rightarrow \infty} B_{n}^{k(n)}(f)= \begin{cases}f & \text { uniformly on } K \text { if } \frac{k(n)}{n} \rightarrow 0, \\ T(f) & \text { uniformly on } K \text { if } \frac{k(n)}{n} \rightarrow+\infty\end{cases}
$$

It is worthy to point out that, under some additional assumptions on $T$, the sequence $\left(B_{n}^{k(n)}(f)\right)_{n \geq 1}(f \in C(K))$ converges uniformly also when $\left.\frac{k(n)}{n} \longrightarrow t \in\right] 0,+\infty[$.

More precisely, if $K$ is a subset of $\mathbf{R}^{d}, d \geq 1$, with non-empty interior and if $T$ maps the subspace of all polynomials of degree $m$ into itself for every $m \geq 1$, then for every $t \geq 0$ there exists a Markov operator $T(t): C(K) \longrightarrow C(K)$ such that for every $f \in C(K)$ and for every sequence $(k(n))_{n \geq 1}$ of positive integers satisfying $\frac{k(n)}{n} \longrightarrow t$,

$$
T(t) f=\lim _{n \rightarrow \infty} B_{n}^{k(n)}(f) \text { uniformly on } K .
$$

Moreover the family $(T(t))_{t \geq 0}$ is a strongly continuous semigroup of operators (briefly, $C_{0^{-}}$ semigroup of operators) whose generator $(A, D(A))$ is the closure of the operator $(Z, D(Z))$ where

$$
D(Z):=\left\{u \in C(K) \mid \lim _{n \rightarrow \infty} n\left(B_{n}(u)-u\right) \text { exists in } C(K)\right\}
$$

and, for every $u \in D(Z) \subset D(A)$

$$
A(u)=Z(u)=\lim _{n \rightarrow \infty} n\left(B_{n}(u)-u\right) \text { uniformly on } K .
$$

Furthermore, $C^{2}(K) \subset D(Z) \subset D(A)$ and for every $u \in C^{2}(K)$

$$
A u(x)=Z u(x)=\frac{1}{2} \sum_{i, j=1}^{d} \alpha_{i j}(x) \frac{\partial^{2} u(x)}{\partial x_{i} \partial x_{i}}
$$

$\left(x=\left(x_{i}\right)_{1 \leq i \leq d}\right)$ where for every $i, j=1, \ldots, d$

$$
\alpha_{i j}(x):=T\left(p r_{i} p r_{j}\right)(x)-x_{i} x_{j} .
$$

The differential operator (4.16) is an elliptic second order differential operator which degenerates on $\partial_{T} K$ (which contains the subset $\partial_{e} K$ of the extreme points of $K$ ).

For more details on the above results and, especially, for the rich theory which is related to them we refer to [3, Chapter 6] and [5, Chapters 4 and 5]. This theory stresses an interesting relationship among positive semigroups, initial-boundary value problems, Markov processes and constructive approximation theory. Some aspects of them will be also treated in the subsequent Sections 5 and 6.

\section{ITERATES AND CONSTRUCTIVE APPROXIMATION OF SEMIGROUPS OF OPERATORS}

Iterates of (positive) linear operators can be usefully involved in the constructive approximation as well as in the qualitative study of (positive) $C_{0}$-semigroups of operators and, hence of the solutions to the initial-boundary value problems governed by them. For a short introduction to the theory of $C_{0}$-semigroups of operators we refer, e.g., to [5, Chapter 2].

We begin by recalling the following results which is a consequence of a more general one due to H. F. Trotter (see [25] or [5, Corollary 2.2.3]).

Theorem 5.6. Let $E$ be a Banach space and let $\left(L_{n}\right)_{n \geq 1}$ be a sequence of bounded linear operators on E. Suppose that 
(i) (stability conditions) there exist $M \geq 1$ and $\omega \in \mathbf{R}$ such that

$$
\left\|L_{n}^{k}\right\| \leq M e^{\omega \frac{k}{n}} \text { for every } k, n \geq 1 .
$$

Furthermore, let $\left(A_{0}, D_{0}\right)$ be a linear operator defined on a dense subspace $D_{0}$ of $E$ and assume that

(ii) (core condition) $\left(\lambda I_{D_{0}}-A_{0}\right)\left(D_{0}\right)$ is dense in $E$ for some $\lambda>\omega$;

(iii) (asymptotic formula) $\lim _{n \rightarrow \infty} n\left(L_{n}(u)-u\right)=A_{0}(u)$ for every $u \in D_{0}$.

Then $\left(A_{0}, D_{0}\right)$ is closable and its closure $(A, D(A))$ is the generator of a $C_{0}$-semigroup $(T(t))_{t \geq 0}$ on $E$ such that

(1) $\|T(t)\| \leq M e^{\omega t}$ for every $t \geq 0$;

(2) $T(t)(f)=\lim _{n \rightarrow \infty} L_{n}^{k(n)}(f)$

for every $f \in E$ and $t \geq 0$ and for every sequence $(k(n))_{n \geq 1}$ of positive integers satisfying $\lim _{n \rightarrow \infty} \frac{k(n)}{n}=$ $t$.

The core condition ( $i i)$ is often difficult to verify; in the special case where $\omega=0$ in $(i)$, i.e., $\left\|L_{n}\right\| \leq 1$, then it can be replaced by

$(i i)^{*}$ there exists a family $\left(E_{i}\right)_{i \in I}$ of finite dimensional subspaces of $D_{0}$ which are invariant under each $L_{n}$ and whose union $\bigcup_{i \in I} E_{i}$ is dense in $E$.

This variant of Trotter theorem is due to R. Schnabl (see [24] or [5, Corollary 2.2.11]). Note also that, because of assumptions $(i)$ and (iii), necessarily

$$
\lim _{n \rightarrow \infty} L_{n}(u)=u \text { for every } u \in E .
$$

i.e., $\left(L_{n}\right)_{n \geq 1}$ is an approximation process on $E$.

In the sequel, a $C_{0}$-semigroup $(T(t))_{t \geq 0}$ which is approximate by a sequence $\left(L_{n}\right)_{n \geq 1}$ as in formula (2) of Theorem 5.6, will be referred to as the limit semigroup associated with the sequence $\left(L_{n}\right)_{n \geq 1}$. Furthermore, a sequence $\left(L_{n}\right)_{n \geq 1}$ verifying conditions $(i),(i i),(i i i)$ (resp. conditions $\left.(i),(i i)^{*},(i i i)\right)$ will be referred to as a Trotter-type admissible sequence (resp. a Schnabl-type admissible sequence).

The generator $(A, D(A)$ of such semigroup will be also referred to as the generator of the sequence $\left(L_{n}\right)_{n \geq 1}$.

Formula (2) of Theorem 5.6 has been successfully and mainly used in order to infer some properties of the sequence $\left(L_{n}\right)_{n \geq 1}$, notably, their saturation properties or, e.g., if $E$ is a Banach function space, converse theorems of convexity (see, e.g., [14], [21], [24], [3, Section 6.1, pp. 420-421] and the references therein).

Starting from the late eighties, we started a long series of investigations, which are also related to these theorems, but we developed a different point of view.

As it is well-known, each $C_{0}$ - semigroup $(T(t))_{t \geq 0}$ of operators on a Banach space $E$ gives rise, indeed, to its infinitesimal generator $A: D(A) \rightarrow E$, which is defined on a dense subspace $D(A)$ of $E$, and to which it corresponds an abstract Cauchy problem, namely

$$
\begin{cases}\frac{d u(t)}{d t}=A u(t) & t \geq 0, \\ u(0)=u_{0} & u_{0} \in D(A) .\end{cases}
$$

When $E$ is a "concrete" continuous function space on a domain $X$ of $\mathbf{R}^{d}, d \geq 1$, the operator $A$ mostly is a differential operator and problem (5.17) turns into an initial-boundary value 
evolution problem

$$
\begin{cases}\frac{\partial u}{\partial t}(x, t)=A(u(\cdot, t))(x) & x \in X, t \geq 0, \\ u(x, 0)=u_{0}(x) & u_{0} \in D(A), x \in X,\end{cases}
$$

the boundary conditions being incorporated in the domain $D(A)$.

Moreover, problem (5.17) (resp. problem (5.18)) has a unique solution if and only if $u_{0} \in D(A)$ and, in such a case, the solution is given by

$$
u(t)=T(t) u_{0}(t \geq 0)
$$

(resp.

$$
\left.u(x, t)=T(t) u_{0}(x) \quad(x \in X, t \geq 0)\right)
$$

and hence, by using the approximation formula (2) of Theorem 5.6,

$$
u(x, t)=T(t)\left(u_{0}\right)(x)=\lim _{n \rightarrow \infty} L_{n}^{k(n)}\left(u_{0}\right)(x),
$$

where the limit is uniform with respect to $x \in X$.

Therefore, if it is possible to determine the operator $A$ and its domain $D(A)$, the initial sequence $\left(L_{n}\right)_{n \geq 1}$ become the key tool to approximate and to study (especially, from a qualitative point of view) the solutions to problems (5.17) or (5.18).

For instance,

- If $H$ is a closed subset of $E$ which is invariant under the operators $L_{n}$, i.e., $L_{n}(H) \subset H$ for every $n \geq 1$, then $T(t)(H) \subset H$ for every $t \geq 0$.

In such a case, this inclusion represents an abstract "spatial regularity results" in the sense that

$$
u(t) \in H \text { for every } t \geq 0 \text { whenever } u_{0} \in H .
$$

or, respectively,

$$
u(\cdot, t) \in H \text { for every } t \geq 0 \text { whenever } u_{0} \in H .
$$

- In several contexts, the approximation formula of the semigroup in terms of iterates of the operators $L_{n}$ allows to determine its asymptotic behaviour, i.e., to determine

$$
\lim _{t \rightarrow+\infty} T(t) u \text { in } E \quad(u \in E)
$$

or, respectively,

$$
\lim _{t \rightarrow+\infty} u(x, t) \quad(u \in E, x \in K)
$$

(see, for instance, the subsequent Theorems 5.7 and 5.8).

- if $E$ is a Banach lattice and each $L_{n}$ is positive, then the semigroup $(T(t))_{t \geq 0}$ is positive; hence $u(t) \geq 0$ for every $t \geq 0$ provided that $u_{0} \geq 0$.

- if $E$ is a Banach lattice and each $L_{n}$ is positive, then

$$
u \leq L_{n}(u) \Longrightarrow u \leq T(s) u \leq T(t) u(u \in E, 0 \leq s \leq t) .
$$

Furthermore, when $E=C(X), X$ compact space, or $E=C_{0}(X), X$ locally compact space, and the operators $L_{n}, n \geq 1$, are positive, then the positive semigroup governs a right-continuous normal Markov process having $X$ as a state space (see, e.g., [3, Section 1.6, pp. 68 - 73]); hence, by means of the operators $L_{n}$, it is also possible to investigate some qualitative properties of the transition functions associated with the Markov process.

Particular attention deserves the important case when the approximating operators are constructively generated by a given positive linear operator $T: C(X) \rightarrow C(X)$ which, in turn, 
allows to determine the differential operator $(A, D(A))$ as well, $X$ being a compact subset of $\mathbf{R}^{d}, d \geq 1$, having non-empty interior.

As a matter of fact, the linear operators generated by such general approach generalize positive approximating operators which are well-known in Approximation Theory, such as Bernstein operators, Kantorovich operators and others ones, and they shed new light on these classical operators and on their usefulness.

Moreover, initial-boundary value evolution problems corresponding to these particular settings, are of current interest as they occur in the study of diffusion problems arising from different areas such as biology, mathematical finance and physics. For more details and for several other aspects related to the above outlined theory, we refer to the monographs [3], [5] and to the recent paper [4].

Below, we show another general property of limit semigroups in the context of $C(X)$ spaces, $X$ compact metric space.

Consider indeed a compact metric space $(X, d)$ and set

$$
\delta(X):=\sup \{d(x, y) \mid x, y \in X\}
$$

and

$$
\operatorname{Lip}(X):=\left\{\left.f \in C(X)|| f\right|_{\text {Lip }}:=\sup _{\substack{x, y \in X \\ x \neq y}} \frac{|f(x)-f(y)|}{d(x, y)}<+\infty\right\} .
$$

We also recall that every Markov operator $T$ on $C(X)$ admits at least one invariant probability measure, i.e., a measure $\mu \in M_{1}^{+}(X)$ such that

$$
\int_{X} T(f) d \mu=\int_{X} f d \mu \text { for every } f \in C(X)
$$

(see, e.g., [20, Section 5.1, p. 178]). Therefore, for every $p \in[1,+\infty[$, from the Hölder inequality it turns out that for every $f \in C(X)$,

$$
\int_{X}|T(f)|^{p} d \mu \leq \int_{X} T\left(|f|^{p}\right) d \mu=\int_{X}|f|^{p} d \mu
$$

and hence $T$ extends to a unique bounded linear operator $T_{p}: L^{p}(X, \mu) \rightarrow L^{p}(X, \mu)$ such that $\left\|T_{p}\right\| \leq 1$. Furthermore, $T_{p}$ is positive as $C(X)$ is a sublattice of $L^{p}(X, \mu)$ and, if $1 \leq p<q<$ $+\infty$, then $T_{p}=T_{q}$ on $L^{q}(X, \mu)$.

From now on, for a given $p \in[1,+\infty$ [, if no confusion can arise, we shall denote by $\widetilde{T}$ the operator $T_{p}$.

In the sequel, given $\mu \in M_{1}^{+}(X)$, we shall denote by $\Lambda(\mu)$ the subset of all Markov operators $T$ on $C(X)$ for which $\mu$ is an invariant measure.

The next result can be found in [11], Corollary 2.5.

Theorem 5.7. Consider a $C_{0}$-semigroup $(T(t))_{t \geq 0}$ of Markov operators and assume that it is the limit semigroup associated with some sequence of Markov operators $\left(L_{n}\right)_{n \geq 1}$. Furthermore, assume that

(i) There exists $\omega \in \mathbf{R}, \omega<0$, such that for every $n \geq 1, L_{n}(\operatorname{Lip}(X)) \subset \operatorname{Lip}(X)$ and $\left|L_{n}(f)\right|_{\text {Lip }} \leq\left(1+\frac{\omega}{n}\right)|f|_{\text {Lip }}$ for every $f \in \operatorname{Lip}(X)$.

(ii) There exists $\mu \in M_{1}^{+}(X)$ such that $L_{n} \in \Lambda(\mu)$ for every $n \geq 1$. Then

(1) For every $n \geq 1$ and $f \in C(X)$

$$
\lim _{m \rightarrow \infty} L_{n}^{m}(f)=\int_{X} f d \mu \quad \text { uniformly on } X
$$


(2) For every $t \geq 0, T(t)(\operatorname{Lip} X) \subset \operatorname{Lip}(X)$ and $|T(t) f|_{\text {Lip }} \leq \exp (\omega t)|f|_{\text {Lip }} \quad(f \in \operatorname{Lip}(X))$. Moreover, each $T(t)$ is invariant under $\mu$, and

$$
\lim _{t \rightarrow+\infty} T(t) f=\int_{X} f d \mu \text { uniformly on } X
$$

for every $f \in C(X)$.

(3) If $1 \leq p<+\infty$, denoting by $\left(\widetilde{L}_{n}\right)_{n \geq 1}$ and $(\widetilde{T}(t))_{t \geq 0}$ the relevant extensions to $L^{p}(X, \mu)$, then $(\widetilde{T}(t))_{t \geq 0}$ is a $C_{0}$-semigroup on $L^{p}(X, \mu)$ and for every $n \geq 1$ and $f \in L^{p}(X, \mu)$,

$$
\lim _{m \rightarrow \infty} \widetilde{L}_{n}^{m}(f)=\int_{X} f d \mu=\lim _{t \rightarrow+\infty} \widetilde{T}(t) f \quad \text { in } \quad L^{p}(X, \mu) .
$$

Remark 5.2. Note that the property of the theorem above which states that each $T(t)$ is invariant under $\mu$, means that

$$
\int_{X} u(x, t) d \mu(x)=\int_{X} u_{0}(x) d \mu(x) \text { for every } u_{0} \in D(A) \text { and } t \geq 0,
$$

$u(x, t)$ being the solution to the initial-boundary value problem governed by the semigroup $(T(t))_{t \geq 0}$.

The next result is also useful to investigate the limit behaviour of the $C_{0}$-semigroups. The proof depends on Corollary 3.2 (when $S=T$ )(for more details see [2, Theorem 2.9] and [23]).

Theorem 5.8. Let $X$ be a compact metric space and consider a Markov projection $T: C(X) \rightarrow C(X)$ such that its range $H:=T(C(X))$ separates the points of $X$. Further, consider $\Phi \in C(X)$ of the form $\Phi=\sum_{n=1}^{\infty} h_{n}^{2}$ as in Theorem 2.1 (with each $h_{n} \in H$ ), so that $\Phi \leq T(\Phi)$ and $\partial_{H} X=\{x \in X \mid$ $T(\Phi)(x)=\Phi(x)\}$.

Let $\left(L_{n}\right)_{n \geq 1}$ be a sequence of Markov operators on $C(X)$ such that $L_{n}(h)=h$ for every $h \in H$ and $n \geq 1$ and set

$$
a_{n, p}:=\max _{x \in X}\left\{T(\Phi)(x)-\Phi(x)-p n\left(L_{n}(\Phi)(x)-\Phi(x)\right)\right\}
$$

$(n \geq 1, p \geq 1)$.

Finally, assume that the sequence $\left(L_{n}\right)_{n \geq 1}$ generates a limit $C_{0}$-semigroup $(T(t))_{t \geq 0}$.

If $\lim _{p \rightarrow \infty} a_{n, p}=0$ uniformly with respect to $n \geq 1$, then

$$
\lim _{t \rightarrow+\infty} T(t)(f)=T(f)
$$

uniformly on $X$ for every $f \in C(X)$.

\section{AN APPlicAtion: BERNSTEIN-DURRMEYER OPERATORS WITH JACOBI WEIGHTS ON THE UNIT HYPERCUBE}

In this section we discuss some applications of the previous results concerning a sequence of Markov linear operators acting on weighted $L^{p}$-spaces on the unit hypercube $Q_{d}:=[0,1]^{d}$, $d \geq 1$, which have been recently introduced and studied in [4].

These operators map weighted $L^{p}$-functions into polynomials on $Q_{d}$ and generalize the Bernstein-Durrmeyer operators with Jacobi weights on [0,1] ([13], [22]).

Although we are mainly interested in the role which they play in the approximation of the corresponding limit semigroups, it seems that they also have an interest on their own as an approximation sequence for continuous functions as well as for weighted $L^{p}$-functions. 
We begin by fixing some additional notation. Let $\gamma=\left(\gamma_{1}, \ldots, \gamma_{d}\right) \in \mathbf{R}^{d}, d \geq 1$. If $x=\left(x_{1}, \ldots, x_{d}\right) \in \mathbf{R}^{d}$, with $x_{i}>0$ for every $i=1, \ldots, d$, we set

$$
x^{\gamma}:=\prod_{i=1}^{d} x_{i}^{\gamma_{i}}
$$

For $x=\left(x_{1}, \ldots, x_{d}\right), y=\left(y_{1}, \ldots, y_{d}\right) \in \mathbf{R}^{d}$, we write $x \leq y$ if $x_{i} \leq y_{i}$ for every $i=1, \ldots, d$. Let $j=\left(j_{1}, \ldots, j_{d}\right), k=\left(k_{1}, \ldots, k_{d}\right) \in \mathbf{N}^{d}$ be two multi-indices such that $k \leq j$; we set

$$
\left(\begin{array}{l}
j \\
k
\end{array}\right):=\prod_{i=1}^{d}\left(\begin{array}{l}
j_{i} \\
k_{i}
\end{array}\right)
$$

From now on we fix $a=\left(a_{1}, \ldots, a_{d}\right), b=\left(b_{1}, \ldots, b_{d}\right) \in \mathbf{R}^{d}$ with $a_{i}>-1$ and $b_{i}>-1$ for all $i=1, \ldots, d$.

Let us denote by $\mu_{a, b} \in M_{1}^{+}\left(Q_{d}\right)$ the absolutely continuous measure with respect to the BorelLebesgue measure $\lambda_{d}$ on $Q_{d}$ with density the normalized Jacobi weight

$$
w_{a, b}(x):=\frac{x^{a}(1-x)^{b}}{\int_{Q_{d}} y^{a}(1-y)^{b} d y} \quad\left(x \in \stackrel{\circ}{Q}_{d}=\right] 0,1\left[^{d}\right) .
$$

Moreover, for every $n \geq 1$, consider the operator $M_{n}: L^{1}\left(Q_{d}, \mu_{a, b}\right) \rightarrow C\left(Q_{d}\right)$ defined by setting, for every $f \in L^{1}\left(Q_{d}, \mu_{a, b}\right)$ and $x \in Q_{d}$,

$$
M_{n}(f)(x):=\sum_{\substack{h \in \mathbf{N}^{d} \\
0_{d} \leq h \leq n_{d}}} \omega_{n_{d}, h}(f)\left(\begin{array}{c}
n_{d} \\
h
\end{array}\right) x^{h}\left(1_{d}-x\right)^{n_{d}-h},
$$

where, for every $n \geq 1$ and $h=\left(h_{1}, \ldots h_{d}\right) \in \mathbf{N}^{d}, 0_{d} \leq h \leq n_{d}$,

$$
\begin{aligned}
& \omega_{n_{d}, h}(f):=\frac{1}{\int_{Q_{d}} y^{h+a}\left(1_{d}-y\right)^{n_{d}-h+b} d y} \int_{Q_{d}} y^{h}\left(1_{d}-y\right)^{n_{d}-h} f(y) d \mu_{a, b}(y) \\
& =\prod_{i=1}^{d} \frac{\Gamma\left(n+a_{i}+b_{i}+2\right)}{\Gamma\left(h_{i}+a_{i}+1\right) \Gamma\left(n-h_{i}+b_{i}+1\right)} \int_{Q_{d}} y^{h+a}\left(1_{d}-y\right)^{n_{d}-h+b} f(y) d y,
\end{aligned}
$$

$\Gamma(u)(u \geq 0)$ being the classical Euler Gamma function.

Clearly, the restriction of each $M_{n}$ to $C\left(Q_{d}\right)$ is a Markov operator on $C\left(Q_{d}\right)$.

As we shall see, these operators are closely related to the degenerate second-order elliptic differential operator defined by

$$
A(u)(x)=\sum_{i=1}^{d} x_{i}\left(1-x_{i}\right) \frac{\partial^{2} u}{\partial x_{i}^{2}}(x)+\left(a_{i}+1-\left(a_{i}+b_{i}+2\right) x_{i}\right) \frac{\partial u}{\partial x_{i}}(x)
$$

for every $u \in C^{2}\left(Q_{d}\right)$ and $x=\left(x_{1}, \ldots, x_{d}\right) \in Q_{d}$.

Operators similar to (6.27) arise in the theory of Fleming-Viot processes applied to some models of population dynamics which, however, usually take places in the framework of $d$ dimensional simplices.

Due to their intrinsic interest, more recently an increasing attention has been turned to them also in the setting of hypercubes as well. The difficulties in studying them lie in the fact that they degenerate on the boundary of $Q_{d}$, which is not smooth because of the presence of sides and corners. 
The next result shows that operator (6.27) is the pregenerator of a Markov semigroup on $C\left(Q_{d}\right)$ and of a positive contraction semigroup in $L^{p}\left(Q_{d}, \mu_{a, b}\right)$; moreover, both these semigroups are the limit semigroups associated with the operators $M_{n}$.

Theorem 6.9. The differential operator $\left(A, C^{2}\left(Q_{d}\right)\right)$ defined by (6.27) is closable and the sequence $\left(M_{n}\right)_{n \geq 1}$ is a Schnabl-type admissible sequence (with $D_{0}=C^{2}\left(Q_{d}\right)$ ), whose limit semigroup is generated by the closure $(B, D(B))$ of $\left(A, C^{2}\left(Q_{d}\right)\right)$.

Moreover, $\mathbb{P}_{\infty}:=\bigcup_{m=1}^{\infty} \mathbb{P}_{m}$, and hence $C^{2}\left(Q_{d}\right)$, is a core for $(B, D(B))$ and $T(t)\left(\mathbb{P}_{m}\right) \subset \mathbb{P}_{m}$ for every $t \geq 0$ and $m \geq 1$, where $\mathbb{P}_{m}$ denotes the linear subspace generated by the polynomials on $Q_{d}$ of degree $\leq m$.

Considering the measure $\mu_{a, b} \in M_{1}^{+}\left(Q_{d}\right)$ with density the normalized Jacobi weight $w_{a, b}(x)\left(x \in \stackrel{\circ}{Q}_{d}\right)$, then, for every $f \in C\left(Q_{d}\right)$ and $n \geq 1$,

$$
\lim _{t \rightarrow+\infty} T(t)(f)=\lim _{m \rightarrow \infty} M_{n}^{m}(f)=\int_{Q_{d}} f d \mu_{a, b}
$$

uniformly on $Q_{d}$, and the measure $\mu_{a, b}$ is the unique invariant measure on $Q_{d}$ for both the sequence $M_{n \geq 1}$ and the semigroup $(T(t))_{t \geq 0}$.

Finally, if $f \in \operatorname{Lip}\left(Q_{d}\right)$, then, for every $n, m \geq 1$ and $t \geq 0$,

$$
\left\|M_{n}^{m}(f)-\int_{Q_{d}} f d \mu_{a, b}\right\|_{\infty} \leq 2\left(1+\frac{\omega}{n}\right)^{m}|f|_{\text {Lip }}
$$

and

$$
\left\|T(t)(f)-\int_{Q_{d}} f d \mu_{a, b}\right\|_{\infty} \leq 2 \exp (\omega t)|f|_{\text {Lip }},
$$

where

$$
\omega:=-\min _{1 \leq i \leq d} \frac{a_{i}+b_{i}+2}{a_{i}+b_{i}+3}<0 .
$$

The above Markov semigroup can be extended, indeed, to each $L^{p}\left(Q_{d}, \mu_{a, b}\right)$ space $(1 \leq p<$ $+\infty)$ as the next result shows.

Theorem 6.10. For every $1 \leq p<+\infty$, the semigroup $(T(t))_{t \geq 0}$ on $C\left(Q_{d}\right)$ extends to a unique positive contraction semigroup $\left(T_{p}(t)\right)_{t \geq 0}$ on $L^{p}\left(Q_{d}, \mu_{a, b}\right)$, whose generator is an extension of $(B, D(B))$ to $L^{p}\left(Q_{d}, \mu_{a, b}\right)$ and $\mathbb{P}_{\infty}$ is a core for it. Moreover, if $f \in L^{p}\left(Q_{d}, \mu_{a, b}\right)$ and $(k(n))_{n \geq 1}$ is an arbitrary sequence of positive integers satisfying $\lim _{n \rightarrow \infty} k(n) / n=t$, then

$$
T_{p}(t)(f)=\lim _{n \rightarrow \infty} M_{n}^{k(n)}(f) \quad \text { in } L^{p}\left(Q_{d}, \mu_{a, b}\right) .
$$

Finally, if $f \in L^{p}\left(Q_{d}, \mu_{a, b}\right)$ and $n \geq 1$,

$$
\lim _{t \rightarrow+\infty} T_{p}(t)(f)=\lim _{m \rightarrow \infty} M_{n}^{m}(f)=\int_{Q_{d}} f d \mu_{a, b}
$$

in $L^{p}\left(Q_{d}, \mu_{a, b}\right)$.

Below we discuss some consequences of Theorem 6.9 (for additional details and proofs we refer to [4]).

Consider the abstract Cauchy problem associated with the closure $(B, D(B))$ of $\left(A, C^{2}\left(Q_{d}\right)\right)$

$$
\begin{cases}\frac{\partial u}{\partial t}(x, t)=B(u(\cdot, t))(x) & x \in Q_{d}, t \geq 0, \\ u(x, 0)=u_{0}(x) & u_{0} \in D(B), x \in Q_{d} .\end{cases}
$$


Since $(B, D(B))$ generates a Markov semigroup $(T(t))_{t \geq 0}$, problem (6.29) admits a unique solution $u: Q_{d} \times\left[0,+\infty\left[\longrightarrow \mathbf{R}\right.\right.$ given by $u(x, t)=T(t)\left(u_{0}\right)(x)$ for every $x \in Q_{d}$ and $t \geq 0$. Hence, taking Theorem 6.9 into account, we may approximate such a solution in terms of iterates of the $M_{n}$ 's, namely

$$
u(x, t)=T(t)\left(u_{0}\right)(x)=\lim _{n \rightarrow \infty} M_{n}^{k(n)}\left(u_{0}\right)(x),
$$

where $(k(n))_{n \geq 1}$ is an arbitrary sequence of positive integers satisfying $\lim _{n \rightarrow \infty} k(n) / n=t$, and the limit is uniform with respect to $x \in Q_{d}$.

Note that $B$ coincides with $A$ on $C^{2}\left(Q_{d}\right)$; therefore, if $u_{0} \in \mathbb{P}_{m}(m \geq 1)$, then $u(x, t)$ is the unique solution to the Cauchy problem

$$
\begin{cases}\frac{\partial u}{\partial t}(x, t)=\sum_{i=1}^{d} x_{i}\left(1-x_{i}\right) \frac{\partial^{2} u}{\partial x_{i}^{2}}(x, t)+\left(a_{i}+1-\left(a_{i}+b_{i}+2\right) x_{i}\right) \frac{\partial u}{\partial x_{i}}(x, t) & x \in Q_{d}, \\ u(x, 0)=u_{0}(x) & t \geq 0, \\ & x \in Q_{d},\end{cases}
$$

and

$$
u(\cdot, t) \in \mathbb{P}_{m} \quad \text { for every } t \geq 0 .
$$

Moreover, each $u(\cdot, t), t \geq 0$, has the same integral of $u_{0}$ with respect to the measure $\mu_{a, b}$ and, thanks to formula (6.9),

$$
\lim _{t \rightarrow+\infty} u(x, t)=\int_{Q_{d}} u_{0} d \mu_{a, b}
$$

uniformly w.r.t. $x \in Q_{d}$.

Next, we enlighten other spatial regularity properties of the solution $u(\cdot, t)$ to $(6.29)$, which, however, we state in terms of the semigroup $(T(t))_{t \geq 0}$.

We set

$$
\operatorname{Lip}\left(Q_{d}\right):=\left\{\left.f \in C\left(Q_{d}\right)|| f\right|_{\text {Lip }}:=\sup _{\substack{x, y \in Q_{d} \\ x \neq y}} \frac{|f(x)-f(y)|}{\|x-y\|_{1}}<+\infty\right\}
$$

and, for $M>0$,

$$
\operatorname{Lip}(M, 1):=\left\{f \in \operatorname{Lip}\left(Q_{d}\right)|| f(x)-f(y) \mid \leq M\|x-y\|_{1}\right\},
$$

where $\|\cdot\|_{1}$ is the norm on $\mathbf{R}^{d}$ defined by $\|x\|_{1}:=\sum_{i=1}^{d}\left|x_{i}\right|$, for every $x=\left(x_{1}, \ldots, x_{d}\right) \in \mathbf{R}^{d}$. More generally, given $0<\alpha \leq 1$, we shall denote by $\operatorname{Lip}(M, \alpha)$ the subset of all Hölder continuous functions $f$ on $Q_{d}$ with exponent $\alpha$ and constant $M$, i.e.,

$$
|f(x)-f(y)| \leq M\|x-y\|_{1}^{\alpha} \quad \text { for every } x, y \in Q_{d} .
$$

Theorem 6.11. Let $\omega$ be the constant defined by (6.28). The following statements hold true:

(a) $T(t)\left(\operatorname{Lip}\left(Q_{d}\right)\right) \subset \operatorname{Lip}\left(Q_{d}\right)$ for every $t \geq 0$; moreover, for every $f \in \operatorname{Lip}\left(Q_{d}\right)$ and $t \geq 0$,

$$
|T(t)(f)|_{\text {Lip }} \leq \exp (\omega t)|f|_{\text {Lip }} ;
$$

in particular, if $f \in \operatorname{Lip}(M, 1)$, then, for every $t \geq 0$,

$$
T(t)(f) \in \operatorname{Lip}(M \exp (\omega t), 1) .
$$


(b) For every $f \in C\left(Q_{d}\right), t \geq 0, \delta>0$,

$$
\Omega(T(t)(f), \delta) \leq(1+\exp (\omega t)) \Omega(f, \delta) .
$$

where $\Omega(g, \delta):=\sup \left\{|g(x)-g(y)| \mid x, y \in Q_{d},\|x-y\|_{1} \leq \delta\right\}$ denotes the usual modulus of continuity ( $g: Q_{d} \rightarrow \mathbf{R}$ bounded function and $\delta>0$ ).

Moreover, if $M>0$ and $0<\alpha \leq 1$,

$$
T(t)(\operatorname{Lip}(M, \alpha)) \subset \operatorname{Lip}(M \exp (\alpha \omega t), \alpha) \subset \operatorname{Lip}(M, \alpha)
$$

(see (6.35)).

(c) If $f \in C\left(Q_{d}\right)$ is convex with respect to each variable, then so is $T(t)(f)$ for every $t \geq 0$. In particular, if $d=1$ and if $f \in C([0,1])$ is convex, then $T(t)(f)$ is convex for every $t \geq 0$.

\section{AN INVITATION FOR FURTHER RESEARCHES}

The above outlined theory shows that, generally speaking, some sequences of positive linear operators can be fruitfully used not only as approximation processes in various function spaces, but also to approximate and to infer qualitative properties of solutions to initial-boundary value problems.

On the light of this second aspect, it seems to be not devoid of interest to look at a kind of inverse problem, namely, given a (degenerate) second-order elliptic differential operator

$$
A_{0}(u)(x)=\frac{1}{2} \sum_{i, j=1}^{d} \alpha_{i j}(x) \frac{\partial^{2} u}{\partial x_{i} \partial x_{j}}(x)+\sum_{i=1}^{d} \beta_{i}(x) \frac{\partial u}{\partial x_{i}}(x)
$$

$\left(u \in C^{2}(X), \alpha_{i j}, \beta_{i} \in C(X), x \in X\right)$ with $X$ subset of $\mathbf{R}^{d}, d \geq 1$, having non-empty interior, try to investigate whether it is possible to construct a suitable Trotter-Schnabl type approximation sequence in a suitable Banach function space such that the closure of $\left(A_{0}, C^{2}(X)\right)$ (if it exists) is the relevant generator.

In order to reach this purpose, it might be fully justified to modify well-know classical approximation processes as well as to try to generalize them introducing more general methods of construction such as, for instance, those which arise from a Markov operator ([3], [5], [6], [7]), even though such last methods seem to have an own intrinsic theoretical interest.

Toward the above mentioned direction several researches have been already developed both in one dimensional contexts (bounded and unbounded interval) and in multidimensional contexts, but it seems that there are still new and interesting cases worthy to be investigated. For more details we refer, e.g., to [8], [9] and the references therein.

\section{REFERENCES}

[1] F. Altomare, Korovkin-type Theorems and Approximation by Positive Linear Operators, Surveys in Approximation Theory, Vol. 5, 2010, 92-164, free available on line at http: / / www.math.technion.ac.il/sat/papers/13/, ISSN 1555578X.

[2] F. Altomare, On some convergence criteria for nets of positive operators on continuous function spaces, J. Math. Anal. Appl. 398 (2013) 542 - 552.

[3] F. Altomare and M. Campiti, Korovkin-Type Approximation Theory and its Applications, de Gruyter Studies in Mathematics 17, Walter de Gruyter, Berlin-New York, 1994.

[4] F. Altomare, M. Cappelletti Montano, V. Leonessa, On the positive semigroups generated by Fleming-Viot type differential operators on hypercubes, Comm. Pure and Appl. Anal., Volume 18, Number 1, January 2019, pp. 323 - 340.

[5] F. Altomare, M. Cappelletti Montano, V. Leonessa and I. Raşa, Markov Operators, Positive Semigroups and Approximation Processes, de Gruyter Studies in Mathematics 61, Walter de Gruyter GmbH, Berlin/Boston, 2014.

[6] F. Altomare, M. Cappelletti Montano, V. Leonessa and I. Raşa, A generalization of Kantorovich operators for convex compact subsets, Banach J. Math. Anal. 11(2017), no. 3, 591 - 614. 
[7] F. Altomare, M. Cappelletti Montano, V. Leonessa and I. Raşa, Elliptic differential operators and positive semigroups associated with generalized Kantorovich operators, J. Math. Anal. Appl. 458(2018), 153 - 173.

[8] F. Altomare and V. Leonessa, An invitation to the study of evolution equations by means of positive linear operators, Lecture Notes of Seminario Interdisciplinare di Matematica, Volume VIII, 1-41, Lect. Notes Semin. Interdiscip. Mat., 8, Semin. Interdiscip. Mat., Potenza 2009.

[9] F. Altomare, V. Leonessa and S. Milella, Bernstein-Schnabl operators on noncompact real intervals, Jaen J. Approx., 1(2) (2009), 223-256.

[10] F. Altomare, V. Leonessa and I. Raşa, On Bernstein-Schnabl operators on the unit interval, Z. Anal. Anwend. 27(2008), no. 3, 353 - 379.

[11] F. Altomare and I. Raşa, Lipschitz contractions, unique ergodicity and asymptotics of Markov semigroups,Bollettino U.M.I. (9) V(2012),1-17.

[12] H. Bauer, Measure and Integration Theory, de Gruyter Studies in Mathematics 26, Walter de Gruyter GmbH, Berlin/Boston, 2011.

[13] H. Berens and Y. Xu, On Bernstein-Durrmeyer polynomials with Jacobi weights, in: C. K. Chui (Ed.), Approximation Theory and Functional Analysis, Academic Press, Boston, 1991, 25-46.

[14] P. L. Butzer and H. Berens, Semi-groups of Operators and Approximation, Springer-Verlag, New York, 1967.

[15] I. Gavrea and M. Ivan, On the iterates of positive linear operators preserving the affine functions, J. Math. Anal. Appl.372(2010),366-368.

[16] I. Gavrea and M. Ivan, Asymptotic behaviour of the iterates of positive linear operators, Abstr. Appl. Anal. 2011, Art. ID 670509, $11 \mathrm{pp}$.

[17] I. Gavrea and M. Ivan, On the iterates of positive linear operators, J. Approx. Theory 163 (2011), 1076-1079.

[18] A. Guessab and G. Schmeisser, Two Korovkin-type theorems in multivariate approximation, Banach J. Math. Anal. 2 (2008), no. 2,121-128.

[19] W. Heping, Korovkin-type theorem and application, J. Approx. Theory, 132 (2005), no. 2, 258-264.

[20] U. Krengel, Ergodic Theorems, de Gruyter Studies in Mathematics 6, W. de Gruyter, Berlin, New York, 1985.

[21] C. A.Micchelli, The saturation class and iterates of the Bernstein polynomials, J. Approx. Theory 8 (1973), 1-18.

[22] R. Paltanea, Sur un opérateur polynomial défini sur l'ensemble des fonctions intégrables, Univ. Babeş-Bolyai, ClujNapoca, 83-2 (1983), 101-106.

[23] I. Raşa, Asymptotic behaviour of iterates of positive linear operators, Jaen J. Approx. 1 (2) (2009), 195-204.

[24] R. Schnabl, Zum globalen Saturationsproblem der Folge der Bernsteinoperatoren, Acta Sci. Math. (Szeged) 31 (1970), 351-358.

[25] H. F. Trotter, Approximation of semigroups of operators, Pacific J. Math. 8 (1958), 887-919.

DipARTiMENTO Di MATEMATICA,

UNIVERSITÀ DEGLI STUDI DI BARI ALDO MORO,

CAMPUS UniVERSITARIO, Via E. ORABONA N. 4,

70125-BARI, ITALY.

E-mail address: francesco.altomare@uniba.it 